\title{
BMJ Open Association between presenteeism and health-related quality of life among Japanese adults with chronic lower back pain: a retrospective observational study
}

Toshinaga Tsuji, ${ }^{1}$ Ko Matsudaira, ${ }^{2}$ Hiroki Sato, ${ }^{1}$ Jeffrey Vietri, ${ }^{3}$ Dena H Jaffe ${ }^{4}$

To cite: Tsuji T, Matsudaira K, Sato $\mathrm{H}$, et al. Association between presenteeism and health-related quality of life among Japanese adults with chronic lower back pain: a retrospective observational study. BMJ Open 2018;8:e21160. doi:10.1136/ bmjopen-2017-021160

- Prepublication history for this paper is available online To view these files, please visit the journal online (http://dx.doi. org/10.1136/bmjopen-2017021160).

Received 16 December 2017 Revised 14 March 2018 Accepted 13 April 2018

\section{Check for updates}

${ }^{1}$ Medical Affairs Department, Shionogi \& Co., Ltd, Osaka, Japan

${ }^{2}$ Department of Medical Research and Management for Musculoskeletal Pain, 22nd Century Medical and Research Center, The University of Tokyo, Tokyo, Japan

${ }^{3}$ Health Outcomes Practice, Kantar Health, Horsham,

Pennsylvania, USA

${ }^{4}$ Health Outcomes Practice, Kantar Health, Tel Aviv, Israel

Correspondence to

Dr Dena H Jaffe;

dena.jaffe@kantarhealth.com

\section{ABSTRACT}

Objectives This study investigated the relationship between presenteeism and health-related quality of life (HRQoL) among Japanese adults with chronic lower back pain (CLBP).

Design This was a retrospective, cross-sectional study. Setting Data were collected via a self-administered online survey of the Japanese adult general population.

Participants The present study used 2014 Japan National Health and Wellness Survey (NHWS) data $(n=30000)$. Specifically, data were included from NHWS respondents who self-reported being employed in the past week and having experienced LBP in the past month, with these symptoms lasting for at least 3 months $(n=239) .84$ $(35.1 \%)$ participants in this study were female.

\section{Primary and secondary outcome}

measures Presenteeism and HRQoL were measured using the Work Productivity and Activity Impairment Questionnaire-General Health (categorical (none: 0\%, low: $10 \%-20 \%$, high: $\geq 30 \%$ ) and continuous) and Medical Outcomes Study 36-Item Short Form Health Survey, respectively. Covariates included patient demographics, health characteristics, pain characteristics and depression severity (Patient Health Questionnaire).

Results Presenteeism was reported by $77.4 \%$ of respondents. High (vs no) presenteeism related to more severe pain in the prior week $(4.9 \pm 2.2$ vs $3.6 \pm 2.1$, $p=0.001)$ and currently $(5.1 \pm 2.1$ vs $3.9 \pm 3.9, p=0.007)$, more pain sites $(1.9 \pm 1.6$ vs $1.1 \pm 1.4, p=0.004)$ and greater depression severity $(7.5 \pm 6.5$ vs $3.6 \pm 3.6, p<0.001)$. Adjusting for covariates, high (vs no) presenteeism related to lower mental and physical HRQoL. For low versus no presenteeism, significant HRQoL differences were observed in general health $(43.0,95 \% \mathrm{Cl} 40.3$ to 45.6 vs $46.9,95 \% \mathrm{Cl} 43.9$ to $49.8, \mathrm{p}=0.015)$.

Conclusions Most respondents experienced presenteeism. Those with high or low presenteeism had poorer HRQoL than respondents with no presenteeism. Monitoring presenteeism rates may help identify workers with an unmet need for better CLBP-related pain management.

\section{INTRODUCTION}

Lower back pain (LBP) is one of the most common forms of musculoskeletal pain, with estimates of lifetime prevalence ranging

\section{Strengths and limitations of this study}

- Focusing on presenteeism, rather than absenteeism as an indicator of pain may have permitted for a more accurate estimate of the relationship between work productivity impairment and health-related quality of life.

- Patient-reported outcome data provide important insights into the humanistic burden of chronic lower back pain.

- All data were self-reported and could not be independently verified.

- Self-selection bias may have reduced the representativeness of the study sample.

from $39 \%$ to $83 \% .{ }^{12}$ LBP is also the leading cause of years lived with disability globally. ${ }^{3}$ Approximately, $20 \%$ of patients with acute LBP continue to experience symptoms for 3 months or more and are subsequently diagnosed with chronic LBP (CLBP), ${ }^{4}$ per both Japanese and American guidelines. ${ }^{5}{ }^{6}$ Exercise and patient education have been recommended for the prevention of LBP. ${ }^{7}$

CLBP is associated with significant disability and impairments in health-related quality of life (HRQoL), as well as with reductions in overall functioning and productivity. ${ }^{8-11}$ CLBP also impacts an individual's ability to work, and it is responsible for a tremendous economic burden. ${ }^{11-13}$ A recent study demonstrated that greater pain severity is associated with higher work productivity impairment among patients with CLBP in the USA; those with more severe CLBP pain incurred over US\$25000 in annual indirect costs due to work productivity loss. ${ }^{10}$ It is important to consider that patients with CLBP may also be suffering from pain at other sites in addition to the lower back. ${ }^{14}$ Research has shown that a higher number of pain sites is correlated with worse outcomes, including greater absenteeism, healthcare resource use and 
work limitations..$^{15}$ A recent Japanese study also suggested that pain severity is associated with impaired HRQoL, increased psychiatric symptoms, impaired daily activities and greater healthcare resource use. ${ }^{16}$

The association between work productivity and health outcomes is of particular relevance in Japan, where work-related functioning may be influenced by cultural determinants. Hence, the effective prevention and management of pain requires a more nuanced examination of these relationships. Work productivity can be measured both by one's absence from work (ie, absenteeism) and by one's presence at work while experiencing health complaints (ie, presenteeism).${ }^{17}$ Because studies have shown lower rates of absenteeism among patients with musculoskeletal conditions in Japan, relative to other countries, it has been suggested that presenteeism may instead represent a more reliable source of information for evaluating the burden of disease attributed to work impairment. ${ }^{18-20}$ For example, the prevalence of absenteeism due to musculoskeletal symptoms was three times lower in Japan than in the UK. ${ }^{19}$ In contrast, presenteeism is a more common predictor of pain and depression among respondents with LBP, ${ }^{11} 2122$ and it is an important marker for occupational mental health status. ${ }^{23}$ Presenteeism is affected by the nature of the worker's medical condition (eg, chronic, acute, etc), individual factors (eg, personality, work attitudes, etc), and contextual factors (eg, culture, organisational policies, etc). ${ }^{17}$ The patient characteristics and health-related outcomes associated with presenteeism among patients with CLBP in Japan are not well understood. Better clarifying these relationships will be imperative to informing the development of clinical interventions in the workplace. Thus, the objective of the current study was to explore the relationship between work impairment and multiple domains of HRQoL, among employed adults with CLBP in Japan.

\section{METHODS}

\section{Design and setting}

For this retrospective, cross-sectional study, data were obtained from the 2014 Japan National Health and Wellness Survey (NHWS; Kantar Health, New York City, NewYork, USA; www.kantarhealth.com), a large web-based survey of individuals aged 18 or older $(n=30000)$.

\section{Data source and participants}

NHWS respondents are recruited through voluntary survey panels, with sampling stratified by gender and age to reflect the demographic distribution of the Japanese general adult population, as reported in the US Census International Database. ${ }^{24}$ The 2014 Japan NHWS was reviewed by Pearl Institutional Review Board (Indianapolis, Indiana, USA; study number 14-KAN-106) prior to participant recruitment and was found to meet the exemption requirements under US Department of Health and Human Services 45CFR46.101 regulations. Potential respondents for the NHWS were identified through the Lightspeed Research (LSR) general panel. General panel members explicitly agreed to join the LSR panel and subsequently receive periodic invitations to participate in various health and non-health-related online surveys. Panel participants are recruited through a variety of means, including coregistration with other internet panels, e-newsletter campaigns and banner placements among other channels. This recruitment process is not conducted strictly by convenience-an attempt is made to approximate the distribution of the adult population in the country of interest. Further, all respondents view an online informed consent form and indicate their consent to participate prior to responding to the survey.

The NHWS questionnaire included items regarding the experience of different medical conditions, sociodemographic characteristics, as well as health outcome measures. All data in the NHWS were self-reported. To be eligible for inclusion in the current study, NHWS respondents must have reported that they were employed in the past week and experienced LBP in the past month, with these symptoms lasting for at least 3 months $(n=239)$. These symptom criteria were chosen to satisfy Japanese and American guidelines for CLBP diagnosis. ${ }^{56}$ Figure 1 displays the flow of study participants.

\section{Variables}

Demographics and health characteristics

Data on age (in years and $<40,40-49,50-59$ and $\geq 60$ years), gender, employment status (employed or not employed), annual household income $(<¥ 3$ million, ¥3 million to $<¥ 5$ million, $¥ 5$ million to $<¥ 8$ million, $\geq ¥ 8$ million or decline to answer), marital status (married/living with a partner or not married/living with a partner) and level of education (university degree or no university degree) were collected for all respondents. Body mass index (BMI) was calculated from height and weight and reported as: underweight $\left(<18.5 \mathrm{~kg} / \mathrm{m}^{2}\right)$, normal weight $(18.5$ to $<25.0 \mathrm{~kg}$ / $\left.\mathrm{m}^{2}\right)$, overweight $\left(25.0\right.$ to $\left.<30.0 \mathrm{~kg} / \mathrm{m}^{2}\right)$, obese $(\geq 30.0 \mathrm{~kg}$ / $\mathrm{m}^{2}$ ) or decline to answer. Respondents provided data on cigarette smoking (current smoker, former smoker or never smoked), alcohol use (none or any) and vigorous exercise at least 1 day in the past month (yes or no) were also included. Comorbidity burden was assessed via scores on the Charlson Comorbidity Index (CCI) ${ }^{25}$ The CCI weights the presence of the following conditions and then sums the result to calculate total scores: HIV/AIDS, metastatic tumour, lymphoma, leukaemia, any tumour, moderate/severe renal disease, hemiplegia, diabetes, mild liver disease, ulcer disease, connective tissue disease, chronic pulmonary disease, dementia, cerebrovascular disease, peripheral vascular disease, myocardial infarction, congestive heart failure and diabetes with end-organ damage. The greater the total CCI score, the greater the comorbidity burden on the respondent.

Work productivity impairment

Work productivity impairment was assessed using the Work Productivity and Activity Impairment-General 


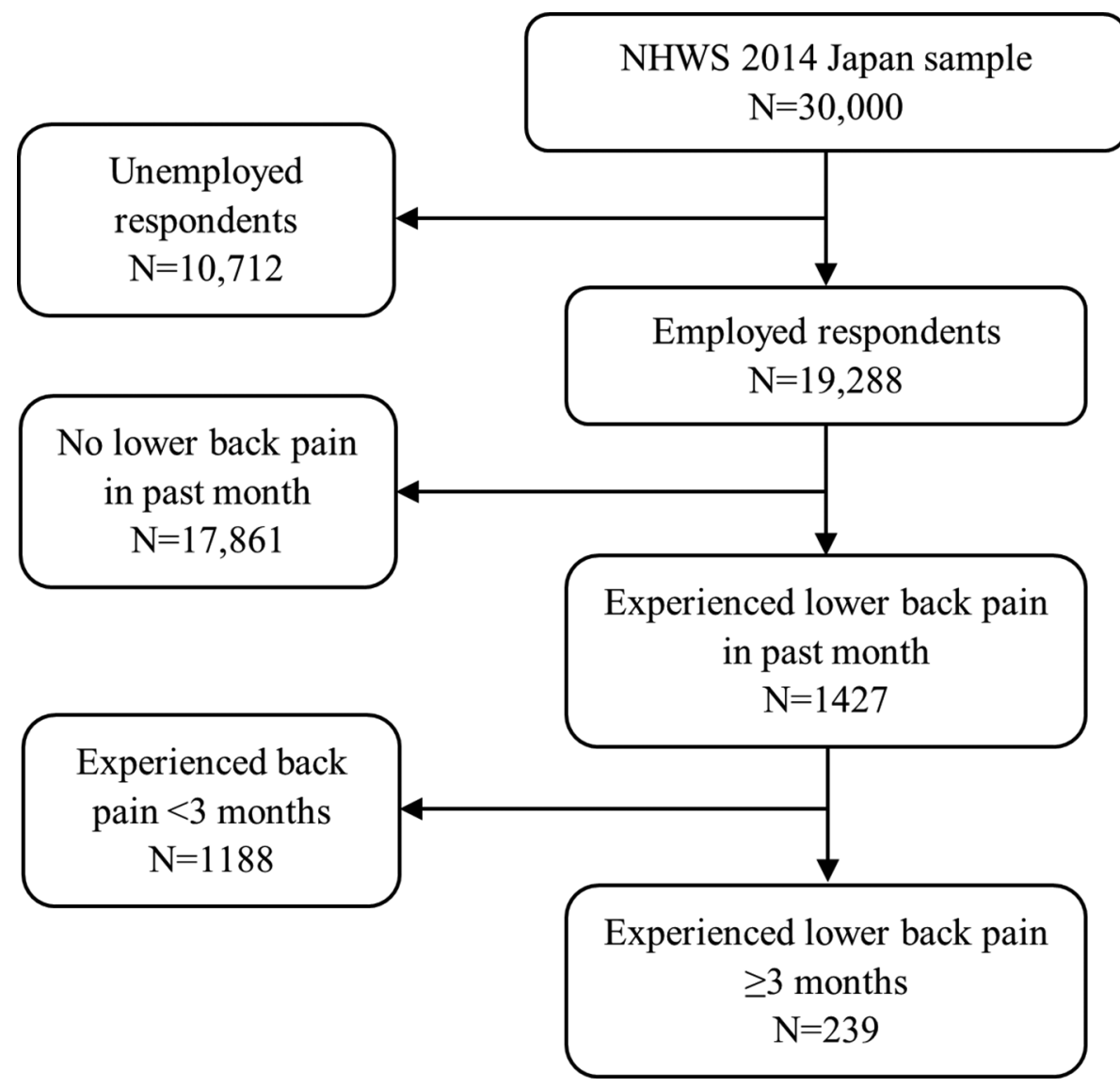

Figure 1 Flow of study participants. NHWS, National Health and Wellness Survey.

Health (WPAI) questionnaire, a six-item validated instrument that consists of four metrics: absenteeism (the percentage of work time missed because of one's health in the past 7 days), presenteeism (the percentage of impairment experienced while at work in the past 7 days because of one's health), overall work productivity loss (an overall impairment estimate that is a combination of absenteeism and presenteeism) and activity impairment (the percentage of impairment in daily activities because of one's health in the past 7 days) ${ }^{26}$ The activity impairment component of the WPAI was not included in this study, although absenteeism, presenteeism and overall work productivity loss were assessed. Scores for absenteeism are calculated by dividing the number of hours missed from work in the past 7 days by the sum of the hours missed from work in the past 7 days and the number of hours worked in the past 7 days. For presenteeism, responses are made on a scale of 1 (health problems had no effect on my work) to 10 (health problems completely prevented me from working) to the following question: 'During the past 7 days, how much did health problems affect your productivity while you were working?' The selected response option is then divided by 10 . Overall work productivity loss is calculated by multiplying presenteeism by the proportion of time worked and adding the product to absenteeism. The values obtained for absenteeism, presenteeism and overall work impairment are then multiplied by 100 to convert the scores to percentages.
The current study primarily focused on presenteeism. Presenteeism was treated as a categorical predictor variable (none: $0 \%$ low: $10 \%-20 \%$ high: $\geq 30 \%$ ), based on a data-driven cut-off representing the median value among respondents reporting any presenteeism. In some analyses, presenteeism was treated as a continuous variable.

Health-related quality of life

HRQoL was measured using the revised Medical Outcomes Study 36-Item Short Form Health Survey (SF-36). ${ }^{27}$ This is a multipurpose, generic HRQoL instrument composed of 36 questions. The SF-36 was designed to report on eight domains of HRQoL (physical functioning, role physical, bodily pain, general health, vitality, social functioning, emotional role limitations and mental health). The version of the SF-36 included in the NHWS has been previously adapted and validated for use with the Japanese adult population. ${ }^{28} 29$ The eight-factor HRQoL profile presented in the current study was based on Japanese population norms, with a mean of 50 and SD of 10; higher scores indicate better HRQoL. ${ }^{30}$ The present analysis also included the SF-36 mental component summary (MCS) and physical component summary (PCS), which were based on US population norms, with a mean of 50 and SD of 10 . Scores on the MCS and PCS can be interpreted, relative to this population average of 50 , as well as with other comparison groups of interest. Higher scores indicate better HRQoL. The SF-36 was also 
used to generate health state utilities on the Short Form-6 dimensions (SF-6D). ${ }^{31}$ The SF-6D is a preference-based single index measure of health state utility using general population values. The SF-6D index has interval scoring properties and yields summary scores on a theoretical 0-1 scale (with an empirical floor of 0.3). Higher index scores indicate better HRQoL.

\section{Pain and treatment characteristics}

Respondents provided data on the severity of their CLBP (mild, moderate or severe). Pain characteristics other than CLBP included pain severity, frequency and duration, as well as the number of pain sites. Pain severity, which was rated using a numerical rating scale anchored by 0 (no pain) and 10 (pain as bad as you can imagine), asked for both current pain, as well as pain in the past week (treated as continuous and categorical: no pain, mild, moderate or severe). The number of additional pain sites was computed as the sum of the pains experienced or diagnosed from among the following pain types: neck pain, shoulder pain, hip pain, joint pain, headache and migraine (treated as continuous and categorical: 0 sites, 1,2 or $\geq 3$ ). Data were also collected on treatment use (yes or no; prescription medication, non-steroidal anti-inflammatory drugs (NSAIDs), over-the-counter medication or herbal products) and the type of physician (yes or no; general internist, gynaecologist, orthopaedist, rheumatologist, pain management specialist or other) who had diagnosed the respondent's CLBP and who had prescribed his/her pain treatment.

\section{Depression symptoms}

The severity of depression symptoms over the last 2 weeks was assessed using the Patient Health Questionnaire (PHQ-9), a validated scale used to screen for depression and assess its severity. ${ }^{32}$ According to the PHQ-9, depression severity is categorised as by scores of $0-4$ (none), 5-9 (mild), 10-14 (moderate), 15-19 (moderately severe) and 20-27 (severe). This scale measures depression through the frequency of anhedonia, depressed mood, sleep disturbance, lack of energy, appetite disturbance, negative self-feelings, difficulty concentrating, psychomotor retardation or agitation and thoughts of self-harm.

\section{Statistical methods}

For all study variables, descriptive statistics were calculated, which included means, SD, median and IQR for continuous variables; frequencies and percentages were computed for categorical variables. Bivariate analyses were also performed for all study variables to examine differences between presenteeism (low or high) and no presenteeism groups in demographics, general health characteristics, pain characteristics, absenteeism and HRQoL, as well as differences in mean presenteeism by category of pain severity and number of pain sites. These analyses included one-way analysis of variance or Kruskal-Wallis non-parametric tests for continuous variables and $\chi^{2}$ or Fisher's exact tests for categorical variables.
Post hoc pairwise comparisons versus no presenteeism were conducted for variables using the Bonferroni correction.

Differences between the presenteeism (low or high) and no presenteeism groups in HRQoL, after adjustment for potential confounds, were examined. Ordinary least squares regression models were used as HRQoL outcomes were normally distribution (data not shown). ${ }^{1321}$ All regression models controlled for demographics and general health characteristics identified a priori, including age, gender, income, education, BMI category, smoking status, alcohol use, exercise activity and CCI. Estimated means and 95\% CIs were reported. For all analyses, two-sided $\mathrm{p}$ values $<0.05$ were considered to be statistically significant.

\section{Patient and public involvement}

Patients and public were not involved in the design or development of the study. No ethical review was undertaken specific to the analysis of the anonymous data presented in this report.

\section{RESULTS}

\section{Descriptive statistics}

The average age of respondents in the full sample $(n=239)$ was $50.9(\mathrm{SD}=13.0)$ years old, and the majority $(64.9 \%)$ was male (table 1). Most respondents $(65.7 \%)$ were married or living with a partner, and slightly more than half $(56.1 \%)$ reported earning a university degree. The majority (65.3\%) had normal BMI and reported drinking alcohol $(80.8 \%)$; approximately one-third $(34.3 \%)$ were current smokers, and almost half $(45.6 \%)$ reported exercising in the past month.

Mean overall work productivity impairment was $32.5 \% \pm 28.7 \%$ for the total sample. The majority $(77.4 \%)$ of respondents reported presenteeism, with an average level of presenteeism of $31.6 \% \pm 28.1 \%$ in the past week. In contrast, absenteeism was rare; it was reported by only $11.7 \%$ of respondents. The mean level of absenteeism in the past 7 days for the total sample was $2.8 \% \pm 11.1 \%$, and it was only reported among those who also reported presenteeism.

The majority of respondents $(91.8 \%)$ reported mild or moderate CLBP (table 2). On a scale of $0-10$, their overall severity of pain was, on average, $4.3 \pm 2.2$ in the prior week, and the current level of pain was rated a mean of 4.5 \pm 2.3 . A large plurality $(40.2 \%)$ reported experiencing pain daily. For most respondents, an orthopaedist had diagnosed $(83.3 \%)$ and prescribed treatment $(70.9 \%)$ for CLBP. Approximately, one-third $(33.1 \%)$ were currently using a prescription medication for pain; of these, $73.4 \%$ used NSAIDs. Use of over-the-counter $(32.2 \%)$ and herbal products $(2.5 \%)$ for pain were also reported. The mean score on the PHQ-9 was 5.5 \pm 5 .6, which indicates mild depression symptoms.

For the total sample, the mean MCS score was 45.0 10.3 , with an average PCS score of $48.2 \pm 6.6$ (table 3 ). The 
Table 1 Sociodemographic and general health characteristics among employed patients with CLBP by level of presenteeism



${ }^{*}$ Continuous variables (eg, age and $\mathrm{CCl}$ ) were compared using a one-way analysis of variance test ( $\mathrm{p}$ values presented) and the KruskalWalllis non-parametric tests. Results from parametric and non-parametric tests were similar. Categorigal variables were compared using $\chi^{2}$ or Fisher's exact test.

†Post hoc pairwise comparisons versus no presenteeism were conducted using the Bonferroni correction. For variables with more than two categories, values in the same row were tested for equality of column proportions. Values with a subscript were significantly different from those with no presenteeism at $p<0.05$.

$\ddagger$ Data were not provided for $n=2$ respondents ( $n=1$ low preseenteeism; $n=1$ high presenteeism).

CLBP, chronic lower back pain.

mean SF-6D health utility index score was $0.68 \pm 0.11$. Average scores on the eight individual domains of the SF-36 ranged from 40.7 (bodily pain) to 46.6 (emotional role limitations).

\section{Bivariate results}

As shown in table 1, the only difference in demographic characteristics to reach statistical significance was for gender. Specifically, respondents with high presenteeism were more likely to be female than respondents with no presenteeism ( $43.9 \%$ vs $18.5 \%, \mathrm{p}<0.05)$.

Those with high presenteeism reported significantly more absenteeism than respondents with no presenteeism $(20.2 \%$ vs $0.0 \%, \mathrm{p}<0.001)$. Relative to those with no presenteeism, respondents with high presenteeism had more severe pain in the prior week $(4.9 \pm 2.2$ vs $3.6 \pm 2.1$, 
Table 2 Pain characteristics of employed patients with CLBP according to levels of presenteeism

\section{Total No presenteeism Low presenteeism High presenteeism}

$(n=239) \quad(0 \%)(n=54) \quad(10 \%-20 \%)(n=71) \quad(\geq 30 \%)(n=114) \quad P$ values ${ }^{*}$

Severity of pain in prior week (0-10)

$\begin{array}{llllll}\text { Mean } \pm \text { SD } & 4.3 \pm 2.2 & 3.6 \pm 2.1 & 3.8 \pm 2.2 & 4.9 \pm 2.2 \dagger & <0.001 \\ \text { Median (IQR) } & 4.0(3) & 3.5(3) & 4.0(4) & 5.0(4) & \end{array}$

Severity of pain currently $(0-10)$

$\begin{array}{llllll}\text { Mean } \pm \text { SD } & 4.5 \pm 2.3 & 3.9 \pm 2.5 & 4.0 \pm 2.3 & 5.1 \pm 2.1 \dagger & 0.001 \\ \text { Median (IQR) } & 5.0(3) & 4.0(3) & 3.0(4) & 5.0(4) & \end{array}$

Duration of lower back pain

(months)

$\begin{array}{llllll}\text { Mean } \pm \text { SD } & 112.7 \pm 120.5 & 112.6 \pm 130.3 & 115.2 \pm 124.6 & 111.2 \pm 113.9 & 0.977 \\ \text { Median (IQR) } & 60.0(156) & 39.0(164) & 60.0(156) & 60.0(128) & \end{array}$

Additional pains (total\#, 0-6)

$\begin{array}{llllll}\text { Mean } \pm \text { SD } & 1.6 \pm 1.6 & 1.1 \pm 1.4 & 1.4 \pm 1.5 & 1.9 \pm 1.6 \dagger & 0.003 \\ \text { Median (IQR) } & 1.0(3) & 1.0(2) & 1.0(2) & 2.0(2) & \end{array}$

Severity of lower back pain $\ddagger$

\begin{tabular}{|c|c|c|c|c|}
\hline Mild (\%) & $114(51.8)$ & $33(66.0)$ & $40(61.5)$ & $41(39.0)$ \\
\hline Moderate (\%) & $88(40.0)$ & $16(32.0)$ & 21 (32.3) & $51(48.6)$ \\
\hline Severe (\%) & $18(8.2)$ & $1(2.0)$ & $4(6.2)$ & $13(12.4)$ \\
\hline
\end{tabular}

Frequency of problems with pain

\begin{tabular}{lllll} 
Daily (\%) & $96(40.2)$ & $19(35.2)$ & $26(36.6)$ & $51(44.7)$ \\
\hline 4-6 times a week (\%) & $38(15.9)$ & $3(5.6)$ & $17(23.9)$ & $18(15.8)$ \\
2-3 times a week (\%) & $52(21.8)$ & $16(29.6)$ & $14(19.7)$ & $22(19.3)$ \\
Once a week (\%) & $19(7.9)$ & $6(11.1)$ & $5(7.0)$ & $8(7.0)$ \\
2-3 times a month (\%) & $21(8.8)$ & $6(11.1)$ & $5(7.0)$ & $10(8.8)$ \\
Once a month or less often (\%) & $13(5.4)$ & $4(7.4)$ & $4(5.6)$ & $5(4.4)$
\end{tabular}

Type of diagnosing doctor for lower back pain

$\begin{array}{lllll}\text { Orthopaedist (\%) } & 199(83.3) & 44(81.5) & 61(85.9) & 94(82.5) \\ \text { Other (\%) } & 40(16.7) & 10(18.5) & 10(14.1) & 20(17.5)\end{array}$

Type of prescribing doctor

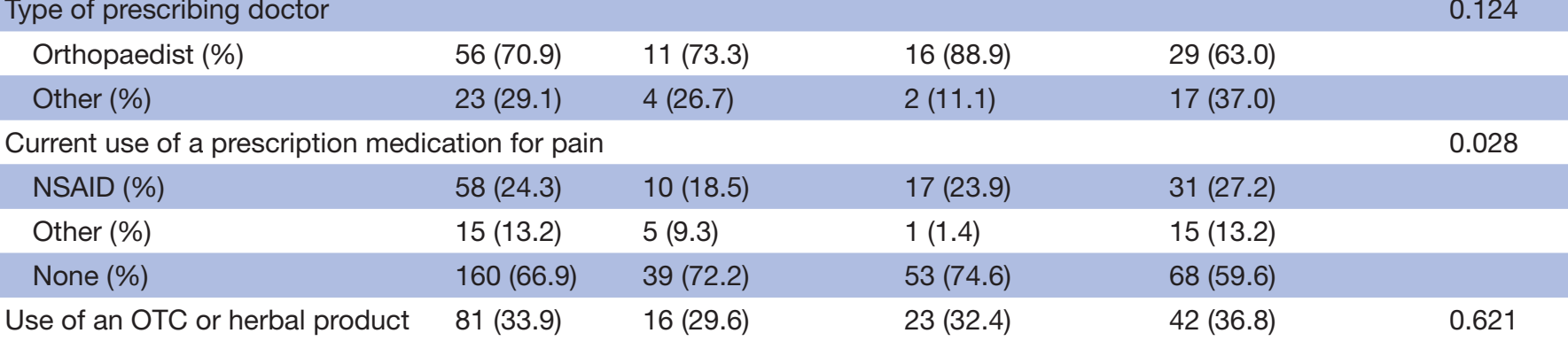

for pain (\%)

\begin{tabular}{|c|c|c|c|c|c|}
\hline $\begin{array}{l}\text { Depression severity } \\
\text { (PHQ-9) }\end{array}$ & & & & & \\
\hline $\begin{array}{l}\text { Mean } \pm \text { SD } \\
\text { Median (IQR) }\end{array}$ & $\begin{array}{l}5.5 \pm 5.6 \\
4.0(7)\end{array}$ & $\begin{array}{l}3.6 \pm 3.6 \\
2(6)\end{array}$ & $\begin{array}{l}3.9 \pm 4.0 \\
3(5)\end{array}$ & $\begin{array}{l}7.5 \pm 6.5 \dagger \\
6.0(7)\end{array}$ & $<0.001$ \\
\hline
\end{tabular}

${ }^{*}$ Continuous variables (eg, severity of pain) were compared using one-way analysis of variance or Kruskal-Walllis non-parametric tests ( $p$ values presented). Results from parametric and non-parametric tests were similar. Categorigal variables were compared using $\chi^{2}$ or Fisher's exact test.

†Post hoc pairwise comparisons versus no presenteeism were conducted using the Bonferroni correction. For variables with more than two categories, values in the same row were tested for equality of column proportions. Values with a subscript were significantly different from those with no presenteeism at $\mathrm{p}<0.05$.

$\neq$ Data were missing for $n=19$ respondents.

CLBP, chronic lower back pain; NSAID, non-steroidal anti-inflammatory drug; OTC, over-the-counter; PHQ-9, Patient Health Questionnaire. 
Table 3 HRQoL (SF-36 summary) outcomes of CLBP respondents according to level of presenteeism

\begin{tabular}{|c|c|c|c|c|c|}
\hline & $\begin{array}{l}\begin{array}{l}\text { Total } \\
(n=239)\end{array} \\
\end{array}$ & $\begin{array}{l}\text { No presenteeism } \\
(0 \%)(n=54) \\
\end{array}$ & $\begin{array}{l}\text { Low presenteeism } \\
(10 \%-20 \%)(n=71)\end{array}$ & $\begin{array}{l}\text { High presenteeism } \\
(\geq 30 \%)(n=114) \\
\end{array}$ & \\
\hline & Mean \pm SD & Mean \pm SD & Mean \pm SD & Mean \pm SD & P values* \\
\hline \multicolumn{6}{|c|}{ HRQoL: summary scores (US norms) } \\
\hline Mental component summary & $45.0 \pm 10.3$ & $50.8 \pm 6.3$ & $48.2 \pm 9.3$ & $40.2 \pm 10.3 \dagger$ & $<0.001$ \\
\hline Physical component summary & $48.2 \pm 6.6$ & $51.0 \pm 5.7$ & $49.9 \pm 5.7$ & $45.7 \pm 6.7 \dagger$ & $<0.001$ \\
\hline Physical functioning & $46.5 \pm 13.0$ & $49.3 \pm 12.3$ & $49.8 \pm 9.9$ & $43.1 \pm 14.2 \dagger$ & 0.001 \\
\hline Physical role limitations & $44.8 \pm 12.6$ & $51.8 \pm 8.7$ & $48.7 \pm 9.3$ & $39.0 \pm 13.5 \dagger$ & $<0.001$ \\
\hline Bodily pain & $40.7 \pm 8.2$ & $44.5 \pm 8.2$ & $42.9 \pm 7.1$ & $37.4 \pm 7.6 \dagger$ & $<0.001$ \\
\hline General health & $43.1 \pm 10.3$ & $49.6 \pm 9.1$ & $45.6 \pm 9.0 \dagger$ & $38.5 \pm 9.5 \dagger$ & $<0.001$ \\
\hline Mental health & $44.3 \pm 10.6$ & $49.6 \pm 8.3$ & $46.9 \pm 10.5$ & $40.3 \pm 10.0 \dagger$ & $<0.001$ \\
\hline
\end{tabular}

*Variables were compared using a one-way analysis of variance test.

†Post hoc pairwise comparisons versus no presenteeism were conducted using the Bonferroni correction and were significantly different from those with no presenteeism at $\mathrm{p}<0.05$.

CLBP, chronic lower back pain; HRQoL; health-related quality of life; SF-6D, Short Form-6 dimension; SF-36, Short Form-36.

$\mathrm{p}<0.05)$ and more severe pain currently $(5.1 \pm 2.1$ vs $3.9 \pm 3.9$, $\mathrm{p}<0.05)$; they were also more likely to report moderate to severe CLBP than those with no presenteeism $(61.0 \%$ vs $34.0 \%, \mathrm{p}<0.05$; table 2 ). Those with high presenteeism also indicated more additional sites of pain than those with no presenteeism $(1.9 \pm 1.6$ vs $1.1 \pm 1.4, \mathrm{p}<0.05)$. High presenteeism was also associated with PHQ-9 scores that were, on average, 3.9 points higher, relative to those with no presenteeism $(7.5 \pm 6.5$ vs $3.6 \pm 3.6, p<0.05)$, indicating greater depression severity for the former than for the latter group. No differences between those with low and no presenteeism in pain characteristics were observed.

Having three or more sites of pain was associated with greater mean presenteeism than having no additional pain sites $(40.5 \% \pm 26.2 \%$ vs $23.5 \% \pm 25.7 \%, \mathrm{p}=0.002)$. There were no differences in presenteeism between those with one $(31.9 \% \pm 30.4 \%, \mathrm{p}=0.088)$ or two $(32.6 \% \pm 28.3 \%$, $\mathrm{p}=0.071)$ pain sites and patients with no additional pain sites. Severe $(45.0 \% \pm 29.7 \%, \mathrm{p}=0.002)$ and moderate $(30.2 \% \pm 25.6 \%, \mathrm{p}=0.043)$ pain in the past week were related to higher average presenteeism than no pain $(13.0 \% \pm 20.0 \%)$. No differences in presenteeism were observed between those with mild pain and those who experienced no pain in the prior week $(27.9 \% \pm 28.1 \%$ vs $13.0 \% \pm 20.0 \%, \mathrm{p}=0.107$ ).

In comparison to those with no presenteeism, respondents with high presenteeism had lower MCS (10.6 points), PCS (5.3 points) and SF-6D scores (0.12 points) (for all, $\mathrm{p}<0.001$; table 4 ). Respondents with high presenteeism also had lower functional health status on all eight SF-36 profile scores, compared with those in the no presenteeism group (for all, $\mathrm{p} \leq 0.001$ ).

\section{Multivariable results}

Results from regression models, adjusting for covariates, were generally consistent with unadjusted comparisons and showed greater HRQoL burden on respondents with high presenteeism, compared with those with no presenteeism, for all SF-36v2 domains $(p<0.001)$, excluding physical functioning (table 4). Respondents with low presenteeism reported lower scores on the general health domain, compared with those with no presenteeism (43.0, $95 \%$ CI 40.3 to 42.6 vs $46.9,95 \%$ CI 43.9 to $49.8, \mathrm{p}=0.015$ ). For the SF-6D health utility index score, no difference was observed for those with low versus no presenteeism; respondents with high presenteeism scored 0.11 points lower than those without presenteeism $(0.72,95 \%$ CI 0.69 to 0.75 vs $0.61,95 \%$ CI 0.66 to 0.63 , $\mathrm{p}<0.001)$.

\section{DISCUSSION}

CLBP is associated with substantial social, economic and humanistic burden globally. The current study sought to extend the understanding of this important health issue through characterisation of employed patients with CLBP in Japan, according to level of presenteeism. Results showed absenteeism was relatively rare, as it was reported by only $12.0 \%$ of working respondents with CLBP. This may be different from other societies, where absenteeism may be a more useful metric for assessing the impact of pain conditions on work productivity. ${ }^{33} 34$ Similarly, the low rate of absenteeism among this sample likely reflects cultural workplace attitudes that have been observed in other studies. ${ }^{121}$ In contrast, presenteeism was reported by approximately four out of five employed respondents 


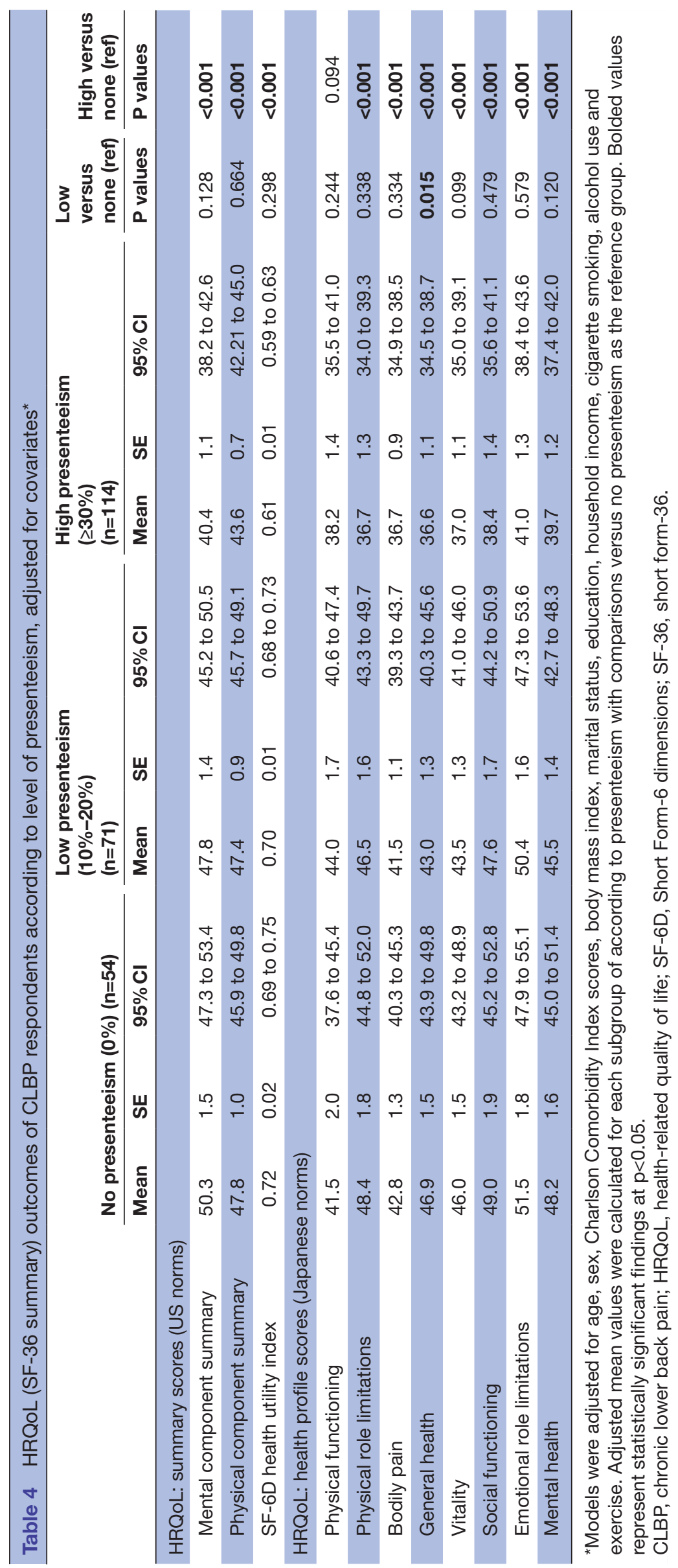


with CLBP in the current study, with the majority losing an estimated $58.0 \%$ of their overall productivity at work in the past week due to their health problems. This is nearly two times as high as the $30.0 \%$ overall work productivity impairment reported by a previous study from the USA. ${ }^{13}$ Thus, by focusing on presenteeism, the current study was able to better evaluate the impact of CLBP on work productivity in the context of the Japanese workforce.

Few differences were observed in sociodemographic characteristics between CLBP respondents with and without presenteeism, although those with high presenteeism were more likely to be female than those with no presenteeism. This finding suggests it may be prudent to focus greater efforts on targeting female workers for health-related interventions in the workplace. Those with high presenteeism also reported higher levels of pain across a number of metrics, including recent and current pain severity. Importantly, those with high presenteeism also reported a greater number of pain sites than those reporting no presenteeism, thus reflecting the possible contribution of multisite pain to work productivity impairment. ${ }^{153536}$ The current study is one of the first to empirically document this phenomenon among Japanese workers.

The negative impact of presenteeism was not confined to work productivity. Respondents with high presenteeism showed impairments in HRQoL, with lower MCS, PCS and SF-6D health utility index scores. Seven of the eight HRQoL dimensions of the SF-36 health profile were also significantly lower among those with high presenteeism, relative to those with no presenteeism.

Collectively, the results were generally consistent with previous research on other disease conditions in Japan. For instance, similar to the scores of respondents with CLBP in this study, Japanese adults with hepatitis $\mathrm{C}$ were found to have mean scores of 46.4 on the MCS and 49.1 on the PCS. ${ }^{37}$ However, overall average work productivity loss among those with hepatitis $\mathrm{C}$, at $19.8 \%$, was considerably lower than the $32.5 \%$ reported by patients with CLBP in the current study. In another prior study, Japanese adults with fibromyalgia actually reported somewhat lower mean scores on the MCS (33.2), PCS (39.2) and SF-6D health utility index $(0.6),{ }^{38}$ relative to respondents with CLBP in the present study. Notably, the rates of absenteeism $(24.5 \%)$, presenteeism $(60.1 \%)$ and overall work productivity impairment $(69.4 \%)$ reported by adults with fibromyalgia were fairly comparable to those observed for respondents with CLBP in the current study.

Overall, the findings of this study have implications for health policy and reinforce the importance of identifying high-risk patients with CLBP by monitoring presenteeism as an indicator of pain and poor HRQoL. In a recent systematic review and meta-analysis, exercise and patient education were shown to be effective in preventing CLBP. ${ }^{7}$ Notably, a recent case-control study showed that over one-third of Japanese adults who self-reported experiencing moderate or severe LBP in the past month were untreated, ${ }^{21}$ which suggests that it may be necessary to improve patients' awareness of and access to treatment to mitigate the impact of CLBP. Employers can also play an integral role in reducing the burden of CLBP. Specifically, certain sources of job stress, such as higher workloads, lower work-life balance and experiencing workplace discrimination, are associated with greater presenteeism $^{39}$; this suggests that employers can potentially decrease presenteeism by modifying work arrangements and by consistently enforcing a zero tolerance policy for discrimination. In light of the prior research and the current study's findings, the industrial health physician in Japan should measure presenteeism, as opposed to absenteeism, to identify patients with CLBP in need of pain management and to introduce pain prevention techniques. In conjunction with these efforts, employers could assign more reasonable workloads, allow flexible scheduling and ensure workers are treated fairly to help reduce presenteeism among those with CLBP. Because the annual indirect costs of work productivity loss for Japanese adults with moderate or severe LBP are nearly double those incurred by matched controls, ${ }^{21}$ addressing presenteeism will be crucial for decreasing the economic burden attributed to this condition.

The results of the current study, including the diagnosis of CLBP and the length of time experiencing pain, were based on self-reported survey data. While the accuracy of these data depend largely on how well the respondents remember their work productivity over the preceding week, the WPAI is a well-established and accepted tool for measuring presenteeism. Self-selection effects may likewise have biased the results, as healthier, younger and/ or wealthier individuals may be more likely to participate in an online study. The sample size of the current study was relatively small, particularly the size of employed respondents with CLBP who reported no presenteeism. A high prevalence of work impairment among respondents with CLBP was observed; however, the small sample size made the statistical tests used in the study less sensitive to potential differences that may actually exist between the groups. Other personal characteristics were not collected in the NHWS, such as physical job demands, which may differ between those with and without presenteeism. The cross-sectional nature of the study precludes causal inferences from being drawn. It is also likely that unmeasured variables, as well as some measured ones (eg, severity of pain), cause both presenteeism and HRQoL. Lastly, analyses did not adjust $\mathrm{p}$ values to account for multiple comparisons, which may have inflated type I error rates. However, it should additionally be noted that almost all $\mathrm{p}$ values for comparisons between high and no presenteeism groups would still be considered statistically significant after a Bonferroni correction.

\section{CONCLUSIONS}

The majority of employed Japanese adults with CLBP reported health-related work impairment, which was largely attributed to high rates of presenteeism. Presenteeism was 
also associated with a host of negative factors in the form of greater pain and depression severity, as well as impairments in most domains of HRQoL. As such, in Japan, presenteeism may be a useful indicator of those workers who have an unmet need for better management of their CLBP pain.

Acknowledgements The authors acknowledge Martine C. Maculaitis for editing assistance on the behalf of Kantar Health, which received funding from Shionogi.

Contributors TT conceived the study idea. JV and DHJ conducted the statistical analyses. TT, KM, HS, JV and DHJ participated in the interpretation of the results and revision of the manuscript for important intellectual content and have read and approved the final version of the manuscript.

Funding This work was supported by Shionogi.

Competing interests All authors have completed the International Committee of Medical Journal Editors (ICMJE) uniform disclosure form and declare the following: TT is a full-time employee and minor stock holder of Shionogi. HS is a full-time employee of Shionogi. KM has received speaking fees from Shionogi, Ayumi Pharmaceutical, Eli Lilly Japan K.K., Ono Pharmaceutical, Pfizer Japan, Nippon Zoki Pharmaceutical, Eisai and Teijin Pharma. KM has received research grants from Pfizer Japan, Eisai, Ayumi Pharmaceutical, Nippon Zoki Pharmaceutical, Ono Pharmaceutical, Lilly Japan K.K., Sumitomo Dainippon Pharma, Astellas Pharma, TOTO and Okamura. KM is a consultant to Shionogi. JV was and DHJ is employed by Kantar Health, which received fees from Shionogi, for access to survey data, analysis and reporting

Patient consent Not required.

Provenance and peer review Not commissioned; externally peer reviewed.

Data sharing statement The dataset used for the current study is not publicly available due to the proprietary nature of the National Health and Wellness Survey, but the subset of data analysed in the current study will be made available from the corresponding author on reasonable request.

Open access This is an open access article distributed in accordance with the Creative Commons Attribution Non Commercial (CC BY-NC 4.0) license, which permits others to distribute, remix, adapt, build upon this work non-commercially, and license their derivative works on different terms, provided the original work is properly cited and the use is non-commercial. See: http://creativecommons.org/ licenses/by-nc/4.0/

(C) Article author(s) (or their employer(s) unless otherwise stated in the text of the article) 2018. All rights reserved. No commercial use is permitted unless otherwise expressly granted.

\section{REFERENCES}

1. Fujii T, Matsudaira K. Prevalence of low back pain and factors associated with chronic disabling back pain in Japan. Eur Spine $J$ 2013;22:432-8.

2. Hoy D, Bain C, Williams $G$, et al. A systematic review of the global prevalence of low back pain. Arthritis \& Rheumatism 2012;64:2028-37.

3. Vos T, Barber RM, Bell B, et al. Global Burden of Disease Study 2013 Collaborators. Global, regional, and national incidence, prevalence, and years lived with disability for 301 acute and chronic diseases and injuries in 188 countries, 1990-2013: a systematic analysis for the Global Burden of Disease Study 2013. Lancet 2015;386:743-800.

4. National Institute of Neurological Disorders and Stroke, National Institutes of Health. Low Back Pain Fact Sheet. https://www.ninds. nih.gov/Disorders/Patient-Caregiver-Education/Fact-Sheets/LowBack-Pain-Fact-Sheet (accessed: May 2017).

5. Qaseem A, Wilt TJ, McLean RM, et al. Clincial Guidelines Committee of the American College of Physicians: Noninvasive treatments for acute, subacute, and chronic low back pain: a clinical practice guideline from the American College of Physicians. Ann Intern Med 2017;166:514-30.

6. Japanese Orthopaedic Association. Clinical practice guideline for the management of low back pain. Tokyo: Nankodo Co., Ltd, 2012.

7. Steffens D, Maher CG, Pereira LS, et al. Prevention of Low Back Pain: A Systematic Review and Meta-analysis. JAMA Intern Med 2016;176:199-208.

8. Gouveia N, Rodrigues A, Eusébio M, et al. Prevalence and social burden of active chronic low back pain in the adult Portuguese population: results from a national survey. Rheumatol Int 2016;36:183-97.

9. Shim JH, Lee KS, Yoon SY, et al. Chronic low back pain in young korean urban males: the life-time prevalence and its impact on health related quality of life. J Korean Neurosurg Soc 2014;56:482-7.

10. Yiengprugsawan V, Hoy D, Buchbinder R, et al. Low back pain and limitations of daily living in Asia: longitudinal findings in the Thai cohort study. BMC Musculoskelet Disord 2017;18:19.

11. Sadosky AB, Taylor-Stokes G, Lobosco S, et al. Relationship between self-reported low-back pain severity and other patientreported outcomes: results from an observational study. J Spinal Disord Tech 2013;26:8-14.

12. Langley $P$, Müller-Schwefe $G$, Nicolaou $A$, et al. The societal impact of pain in the European Union: health-related quality of life and healthcare resource utilization. J Med Econ 2010;13:571-81.

13. McDonald M, DiBonaventura M, Ullman S. Musculoskeletal pain in the workforce: the effects of back, arthritis, and fibromyalgia pain on quality of life and work productivity. J Occup Environ Med 2011;53:765-70.

14. Hartvigsen J, Natvig B, Ferreira M. Is it all about a pain in the back? Best Pract Res Clin Rheumatol 2013;27:613-23.

15. de Fernandes RC, Burdorf A. Associations of multisite pain with healthcare utilization, sickness absence and restrictions at work. Int Arch Occup Environ Health 2016;89:1039-46.

16. Montgomery W, Vietri J, Shi J, et al. The relationship between pain severity and patient-reported outcomes among patients with chronic low back pain in Japan. J Pain Res 2016;9:337-44.

17. Johns G. Presenteeism in the workplace: a review and research agenda. J Organ Behav 2010;31:519-42.

18. Matsudaira $\mathrm{K}$, Konishi $\mathrm{H}$, Miyoshi $\mathrm{K}$, et al. Potential risk factors of persistent low back pain developing from mild low back pain in urban Japanese workers. PLoS One 2014;9:e93924-10.

19. Matsudaira K, Palmer KT, Reading I, et al. Prevalence and correlates of regional pain and associated disability in Japanese workers. Occup Environ Med 2011;68:191-6.

20. Wada K, Arakida M, Watanabe R, et al. The economic impact of loss of performance due to absenteeism and presenteeism caused by depressive symptoms and comorbid health conditions among Japanese workers. Ind Health 2013:51:482-9.

21. Sadosky AB, DiBonaventura M, Cappelleri JC, et al. The association between lower back pain and health status, work productivity, and health care resource use in Japan. J Pain Res 2015;8:119-30.

22. Tsuji T, Matsudaira $\mathrm{K}$, Sato $\mathrm{H}$, et al. The impact of depression among chronic low back pain patients in Japan. BMC Musculoskelet Disord 2016;17:447.

23. Kono Y, Uji M, Matsushima E. Presenteeism among japanese IT employees: personality, temperament and character, job strain and workplace support, and mental disturbance. Psychology 2015;06:1971-83.

24. US Census Bureau, US Census International Database. Mid-Year Population by Single Year Age Groups - Custom Region - Japan. 2014 https://www.census.gov/population/international/data/idb/ region.php? $\mathrm{N}=\% 20$ Results $\% 20 \& \mathrm{~T}=15 \& \mathrm{~A}=$ separate $\& \mathrm{RT}=0 \& \mathrm{Y}=2014 \&$ $\mathrm{R}=-1 \& \mathrm{C}=\mathrm{JA}$ (accessed: May 2017).

25. Charlson ME, Pompei P, Ales KL, et al. A new method of classifying prognostic comorbidity in longitudinal studies: development and validation. J Chronic Dis 1987;40:373-83.

26. Reilly MC, Zbrozek AS, Dukes EM. The validity and reproducibility of a work productivity and activity impairment instrument. Pharmacoeconomics 1993;4:353-65.

27. Maruish ME. User's manual for the SF36v2® Health Survey. 3rd edn. Lincoln: QualityMetric, Inc, 2011.

28. Suzukamo Y, Fukuhara S, Green J, et al. Validation testing of a three-component model of Short Form-36 scores. J Clin Epidemiol 2011;64:301-8.

29. Fukuhara S, Bito S, Green J, et al. Translation, adaptation, and validation of the SF-36 Health Survey for use in Japan. J Clin Epidemiol 1998;51:1037-44.

30. Fukuhara S, Suzukamo Y. Manual of SF-36v2 Japanese version. Kyoto: Institute for Health Outcomes \& Process Evaluation Research, 2004.

31. Brazier J, Roberts J, Deverill M. The estimation of a preferencebased measure of health from the SF-36. J Health Econ 2002;21:271-92.

32. Kroenke K, Spitzer RL, Williams JB. The PHQ-9: validity of a brief depression severity measure. J Gen Intern Med 2001;16:606-13.

33. Mesas AE, González AD, Mesas CE, et al. The association of chronic neck pain, low back pain, and migraine with absenteeism due to health problems in Spanish workers. Spine 2014;39:1243-53.

34. Dibonaventura MD, Gupta S, McDonald M, et al. Impact of self-rated osteoarthritis severity in an employed population: cross-sectional 
analysis of data from the national health and wellness survey. Health Qual Life Outcomes 2012;10:30.

35. Neupane S, Virtanen P, Leino-Arjas P, et al. Multi-site pain and working conditions as predictors of work ability in a 4-year follow-up among food industry employees. Eur J Pain 2013;17:444-51.

36. Phongamwong $\mathrm{C}$, Deema $\mathrm{H}$. The impact of multi-site musculoskeletal pain on work ability among health care providers. $J$ Occup Med Toxicol 2015;10:21.
37. Dibonaventura MD, Yuan Y, Lescrauwaet B, et al. Multicountry burden of chronic hepatitis $\mathrm{C}$ viral infection among those aware of their diagnosis: a patient survey. PLOS One 2014;9:e86070.

38. Lee LK, Ebata N, Hlavacek P, et al. Humanistic and economic burden of fibromyalgia in Japan. J Pain Res 2016;9:967-78.

39. Yang T, Zhu M, Xie X. The determinants of presenteeism: a comprehensive investigation of stress-related factors at work, health, and individual factors among the aging workforce. $J$ Occup Health 2016;58:25-35. 